\title{
Reliability-Aware Heterogeneous 3D Chip Multiprocessor Design
}

\author{
Ismail Akturk • Ozcan Ozturk
}

Received: 2 October 2012 / Accepted: 12 March 2013 / Published online: 4 April 2013

(C) Springer Science+Business Media New York 2013

\begin{abstract}
Ability to stack separate chips in a single package enables three-dimensional integrated circuits (3D ICs). Heterogeneous 3D ICs provide even better opportunities to reduce the power and increase the performance per unit area. An important issue in designing a heterogeneous 3D IC is reliability. To achieve this, one needs to select the data mapping and processor layout carefully. This paper addresses this problem using an integer linear programming (ILP) approach. Specifically, on a heterogeneous 3D CMP, it explores how applications can be mapped onto 3D ICs to maximize reliability. Preliminary experiments indicate that the proposed technique generates promising results in both reliability and performance.
\end{abstract}

Keywords Reliability $\cdot$ Multicore $\cdot 3 D \cdot$ Data mapping

\section{Introduction}

As technology scales, the International Technology Roadmap for Semiconductors (ITRS) projects that the number of cores will drastically increase to satisfy performance requirements of future applications [5]. Once the number of cores passes some threshold (16 cores), conventional pointto-point buses will no longer be a sufficient interconnect structure. These future applications will therefore require a Network-on-Chip (NoC) [14], where a dedicated on-chip

Responsible Editor: H. Manhaeve

I. Akturk · O. Ozturk $(\bowtie)$

Computer Engineering Department, Bilkent University,

Ankara, Turkey

e-mail: ozturk@cs.bilkent.edu.tr

I. Akturk

e-mail: iakturk@cs.bilkent.edu.tr network (with switches and links) is used to perform the communication between cores. NoCs have shown to be able to handle the required communications between the cores in a scalable, flexible, programmable, and reliable fashion [14].

In addition to NoCs, three-dimensional integrated circuit (3D IC) [4] is an attractive option for overcoming the barriers in interconnect scaling. 3D ICs are built using multiple device layers stacked together with a direct tunnel between them, thereby allowing them to reduce the global interconnect. Moreover, 3D ICs provide higher performance and lower power consumption due to the reduced interconnect (wire) length. Other benefits include support for realization of mixed-technology chips, higher packing density, and smaller footprint.

3D NoCs $[6,7,14]$ have been introduced to combine these two techniques (3D ICs and NoCs) to achieve better performance with higher scalability. 3D ICs reduce the global interconnect delay, thereby improve performance. On the other hand, NoCs provide scalable communication framework. While, homogeneous NoCs have been widely used for both 2D and 3D ICs, they are limited compared to their heterogeneous counterparts. This follows from the fact that every application has different processing requirements and memory footprint. A powerful core will be a better match for an application with a high level of instructionlevel parallelism, while a simpler core will be sufficient for applications with lower instruction-level parallelism. Therefore, it is more effective to use heterogeneous NoCs.

As the die size shrinks with the advanced production technology, one of the challenging problems in the context of 3D NoC systems becomes reliability. Reliability of 3D ICs is effected by both temperature and thermo-mechanical stress. This is especially caused by the limited cooling capability between the layers. Specifically, vias become more 
and more sensitive and when the via fails to make proper connection, unwanted loss in yield and decrease in reliability may occur. Reliability for 3D ICs have been explored from different angles $[2,8-11,16]$. Through Silicon Vias (TSVs) are the most recent medium in stacking [8] multiple dies on a 3D IC. However, these vias become more sensitive with higher temperatures that can be caused by more activity or traffic. Since TSVs are bridges between layers, they are potentially more prone to thermal stress. Therefore, reducing the TSV communication load has potential of improving reliability. This work aims at increasing the reliability of an application through effective mapping on 3D heterogeneous IC. Contribution of the approach is in two folds:

- An ILP formulation of the problem of maximizing the reliability of a given application. This is achieved through optimal placement of nodes in a 3D NoC.

- Minimization of the communication cost between the nodes, thereby improving both performance and energy consumption.

ILP-based approach presented here targets at reducing the amount of layer-to-layer communication on TSVs, while keeping the overall communication overheads minimum. The remainder of this paper is structured as follows. Section 2 gives the related work on heterogeneous 3D NoCs and reliability. Section 3 discusses the overview of proposed approach. The details of the ILP (integer linear programming) based formulation are given in Section 4, and an experimental results are presented in Section 5. The paper is concluded in Section 6.

\section{Related Work}

Related work can be summarized in three parts, namely, 3D ICs, 3D NoCs and 3D reliability. 3D technologies and benefits of 3D ICs over 2D ICs have been presented by Davis et al. [4]. Topol et al. [18] review the process steps and design aspects of 3D ICs. Andry et al. [3] discuss a three-dimensional (3D) chip stacking technology using fine-pitched interconnects. Sakuma et al. [15] reviews the 3D integration technologies, including process technology and reliability characterization. Akasaka [1] presents 3D IC technology for fabrication, total power consumption estimation and chip cooling. Zhao et al. [19] studies DC current crowding and its impact on 3D power integrity.

Pavlidis et al. [14] compare 3D NoC over 2D NoC from a physical constraints perspective. These constraints include the maximum number of planes that can be vertically stacked and the asymmetry between the horizontal and vertical communication channels of the network. Li et al. [6] focus on the second level (L2) cache design for 3D architectures. Similarly, Ozturk et al. [13] try to place processor cores and data blocks optimally in a 3D design. Mesochronous communication scheme for 3D NoCs have been explored by Loi et al. [7].

Minz et al. proposed a 3D module and decoupling capacitance (a.k.a decap) placement algorithm that tries to distribute the thermal profile on the circuit evenly and reduce the power noise [10]. The algorithm is trying to find the location of each block in the 3D placement layers without overlap and it tries to minimize the footprint area, total wire length, maximum block temperature, total amount of decap required to suppress simultaneous switching noise (SSN) under the given tolerance value. They showed that there is little correlation between thermal and decap objectives that allows them to optimize these objectives simultaneously. They extended existing 2D sequence pair scheme of Murata et al. [11]. Specifically, each layer has its own sequence pair to represent the relative positions among the blocks in it. Then, they used simulated annealing to search through the solution space using various intra-layer or interlayer moves. Malta et al. discussed the characterization of thermo-mechanical stress and reliability issues for $\mathrm{Cu}$-filled Through Silicon Vias (Cu TSVs) [8]. An X-ray imaging method was used for fast nondestructive analysis of $\mathrm{Cu}$ TSV plating profiles. It was observed that TSVs exposed to increased temperature exhibited a substantial increase in grain size which was associated with the $\mathrm{Cu}$ protrusion effect. Alam et al. developed a framework to enable reliability analysis in 3D circuits called ERNI-3D [2]. It is a Reliability Computer Aided Design (RCAD) tool that is a capable of comparison of 2D and 3D circuit layouts. Similar to study of Alam et al., Shayan et al. proposed a framework to analyze the reliability of 3D power distribution network under local through silicon via failures [17]. The 3D power distribution network is extracted and modeled in frequency domain considering skin effect. The model is first solved in frequency domain to identify the behavior of the system. Then, the time domain voltage noise under worst-case transistor switching currents is obtained with enhanced vector fitting algorithm. The objective of the optimization is to increase the reliability of the 3D structures and reduce the voltage noise while minimizing the block out area from TSV design rules. They showed that the increase in the dimension and density reduce the routable area of the stacked dies. As the width in the tiers increases, the power noise decreases. Selvanayagam et al. worked on thermo-mechanical reliability of through silicon via for different dimensions [16]. The increase in the TSV diameter will increase the thermomechanical strains and as a result the reliability is reduced. They showed the existence of the trade off between the reliability and the power noise reduction as the TSV diameters increases. Minas et al. presented the challenges of and some emerging solutions for 3D processing phases including TSV insertion and wafer thinning [9]. These processes 
have an impact on the functionality, performance and reliability of the circuit. Approach presented in this paper is different from these techniques such that it implements a reliability-aware node/task mapping.

\section{Overview}

High level view of the ILP-based approach is shown in Fig. 1. After necessary parallelization and mapping steps, the input code is fed to the compiler analysis module. The compiler analysis module captures the communication characteristics of processors and they are subsequently used in the ILP solver. Each processor can potentially have different characteristics in terms of performance, energy consumption, area requirement, and communication bandwidth. According to the processor characteristics and communication requirements, processors are laid out on the 3D NoC. Moreover, various constraints such as die area, temperature limit, number of layers, and performance are considered. Locations of processors are selected based on reliability objectives while keeping the communication cost at reasonable levels. Also note that, the objective function can be replaced with a combination of reliability, performance, and energy using different weights.

An example 3D NoC architecture is given in Fig. 2, where multiple layers of heterogeneous processors are connected using network switches/routers represented by $R$. This heterogeneous 3D NoC architecture is exposed to the compiler to enable accesses to the state of the processors, the network switches, and the data/code movements. Note that, heterogeneous processors are represented by $C P U$ and memory hierarchies are represented by $M H$. Each layer of the $3 \mathrm{D} \mathrm{NoC}$ architecture is considered as a grid, where

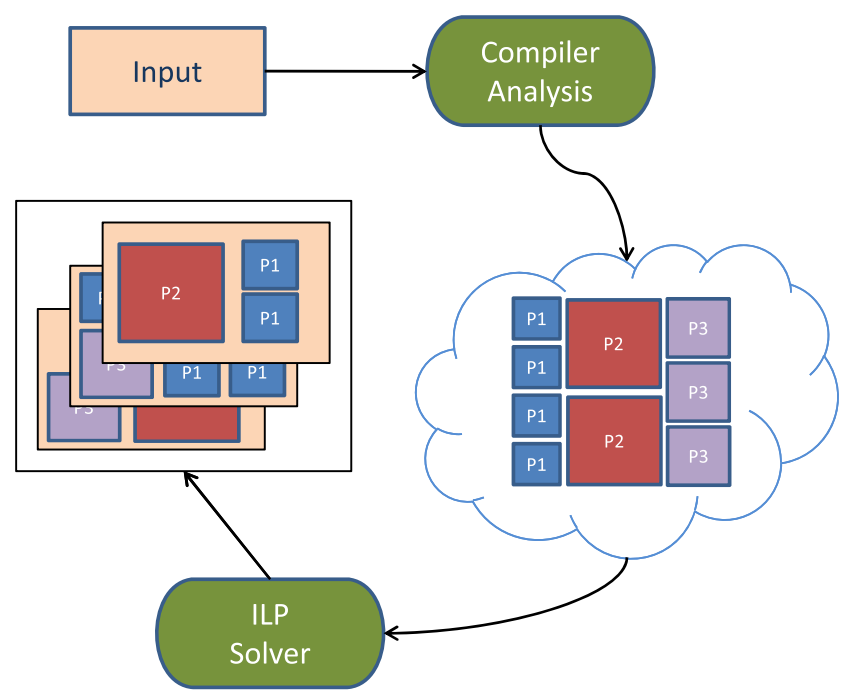

Fig. 1 High level view of the ILP-based approach

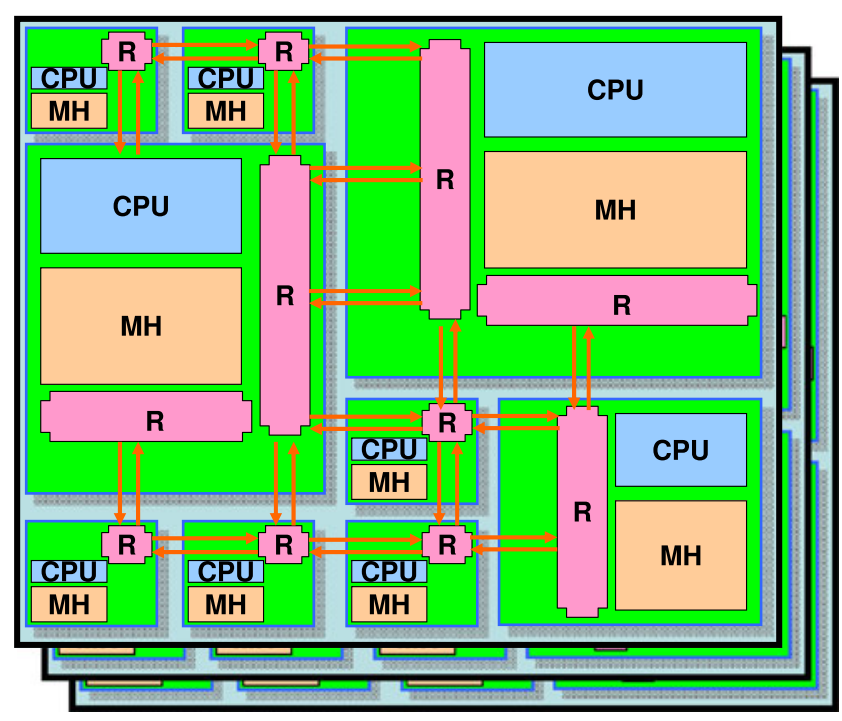

Fig. 2 3D NoC-based CMP architecture

processors are mapped according to their dimensions. Processors are considered to have widths and heights based on the same unit length as the grid. In-layer communication distances are captured using the coordinates of processors in 2D grid space with Manhattan distance. Moreover, interlayer distances include the communication overhead caused by layer-to-layer transmissions.

\section{ILP Formulation}

Integer linear programming (ILP) is an optimization technique which targets optimization of a linear objective function subject to linear function constraints and integer solution variables. A special case of ILP is the $0-1$ ILP, where solution variables are required to be either 0 or 1 . In this context, ILP is used to formulate the reliability problem on a 3D NoC to find the optimal location of each processor. There are two important goals in selecting the location of processors:

- Reduce communication overhead by placing the frequently communicating nodes as close as possible.

- Improve reliability by minimizing the inter-layer communications.

These two goals can potentially contradict with each other when the layer-to-layer communication is considered. This is due to the fact that third dimension provides a lot of opportunities in terms of improving the connectivity of processors. Processors can potentially communicate much faster through TSVs compared to in-layer communications. However, this also results in with increased levels of heat density around vias, making them more and more sensitive. Therefore, ILP aims at reducing the communication 
cost, simultaneously tries not to map high communicating nodes onto separate layers as this increases the use of TSVs which are less reliable compared to the in-layer communication.

This section presents an ILP formulation of the problem of maximizing reliability while minimizing the data communication cost of a given application. This is achieved through optimal placement of nodes in a 3D NoC. While overall ILP formulation has more details, for clarity, we only give the important parts of it. A commercial tool, Xpress-MP [12], is used to formulate and test the ILPbased approach. Xpress-MP takes the problem as a Mosel description which is a plain text file with descriptions of binary variables, constraints, and objective function. Solver (Xpress-MP) generates the output as a plain text file which lists the values of decision variables. Table 1 gives the constant terms and binary variables used in the ILP formulation.

Assuming that 3D NoC chip has dimensions of $D_{X}$ and $D_{Y}$ in the 2D grid space and $L$ number of layers, the ILP problem can be formulated to map the $P$ number of processors on this 3D NoC. Note that, each processor has its own dimensions expressed as $D P X_{p}$ and $D P Y_{p}$. Communication intensity of two processors, namely $p_{1}$ and $p_{2}$, is given

Table 1 The constant terms and binary variables used in the ILP formulation

\begin{tabular}{ll}
\hline Constant & Definition \\
\hline$P$ & Number of processors \\
$D_{X}, D_{Y}$ & Dimensions of the 2D grid \\
$L$ & Number of layers in 3D NoC \\
$D P X_{p}, D P Y_{p}$ & Dimensions of processor $p$ \\
$I_{p_{1}, p_{2}}$ & Communication intensity of processors \\
$\phi$ & $p_{1}$ and $p_{2}$ \\
& In-layer vs. layer-to-layer communication \\
Variable & cost ratio \\
Loc $(p)_{x, y}^{l}$ & Definition \\
Occ $(p)_{x, y}^{l}$ & Processor $p$ is in $(x, y)$ coordinates \\
& on layer $l$ \\
In-layer $\left(p_{1}, p_{2}\right)_{d}$ & Processor $p$ occupies $(x, y)$ coordinates \\
Inter-layer $\left(p_{1}, p_{2}\right)_{l}$ & on layer $l$ \\
Comm & Manhattan distance between processors \\
Comm & $p_{1}$ and $p_{2}$ is $d$ \\
Comm & Layer-to-layer distance between processors \\
\hline & $p_{1}$ and $p_{2}$ is $l$ \\
& Total in-layer communication \\
& Total layer-to-layer communication \\
& Total communication \\
\hline
\end{tabular}

These are either architecture specific or program specific. $L$ indicates the number of layers in the 3D chip with $I_{p_{1}, p_{2}}$. As mentioned before, using TSVs has contradicting effects. A weighted objective function is considered to capture the potential effects on reliability and overall communication. This is achieved by the $\phi$ constant which is used as a knob for choosing in-layer versus layer-to-layer communication.

In the ILP formulation, location of processor $p$ is captured by $\operatorname{Loc}(p)_{x, y}^{l}$, where,

- $\operatorname{Loc}(p)_{x, y}^{l}$ : indicates whether processor $p$ is in $(x, y)$ coordinates in the 2D grid space and on the $l$ layer.

Since a processor can potentially occupy multiple unit spaces in the 2D grid space, a $0-1$ variable named as $O c c(p)_{x, y}^{l}$ is introduced. This binary variable will depend on the dimensions of the processor given with $D P X_{p}, D P Y_{p}$.

- $O c c(p)_{x, y}^{l}$ : indicates whether processor $p$ occupies $(x, y)$ coordinates of the $l$ layer.

Two binary variables have been introduced to capture the distances between two processors; In-layer and Inter-layer. Specifically,

- In-layer $\left(p_{1}, p_{2}\right)_{d}$ : indicates whether the Manhattan distance in 2D grid space between processors $p_{1}$ and $p_{2}$ is equal to $d$.

- Inter-layer $\left(p_{1}, p_{2}\right)_{l}$ : indicates whether the layer-tolayer distance between processors $p_{1}$ and $p_{2}$ is equal to $l$.

In addition to the specified binary variables, there are also non-binary variables to capture different values in the optimization problem. However, these variables are not given here for simplicity. These binary and non-binary variables are used in satisfying various constraints, first of which is one-to-one mapping between processor and 2Dgrid coordinate system at the specified layer the processor is in.

$\sum_{x=1}^{D_{X}} \sum_{y=1}^{D_{Y}} \sum_{l=1}^{L} \operatorname{Loc}(p)_{x, y}^{l}=1, \quad \forall p \in(1, P)$.

To ensure one-to-one mapping, processor needs to be assigned a single coordinate, where $x$ and $y$ indicate the 2Dgrid coordinates, whereas $l$ indicates the layer. Similarly, a specific coordinate on every layer can only be mapped to a single processor which is captured by:

$\sum_{p=1}^{P} \operatorname{Occ}(p)_{x, y}^{l}=1, \forall x \in\left(1, D_{X}\right), \forall y \in\left(1, D_{Y}\right), \forall l \in(1, L)$. 
As mentioned earlier, total data communication requirement at a certain layer is estimated by using the Manhattan distance on a $2 \mathrm{D}$-grid space.

$$
\begin{aligned}
\operatorname{In-layer}\left(p_{1}, p_{2}\right)_{d} & \geq \operatorname{Loc}\left(p_{1}\right)_{x_{1}, y_{1}}^{l_{1}}+\operatorname{Loc}\left(p_{2}\right)_{x_{2}, y_{2}}^{l_{2}}-1, \\
d & =\left|x_{1}-x_{2}\right|+\left|y_{1}-y_{2}\right| .
\end{aligned}
$$

On the other hand, inter-layer communication distance can be captured using the layers the two processors are in:

$$
\begin{aligned}
\operatorname{Inter-layer}\left(p_{1}, p_{2}\right)_{l} & \geq \operatorname{Loc}\left(p_{1}\right)_{x_{1}, y_{1}}^{l_{1}}+\operatorname{Loc}\left(p_{2}\right)_{x_{2}, y_{2}}^{l_{2}}-1, \\
l & =\left|l_{1}-l_{2}\right| .
\end{aligned}
$$

Total communication load within 2D layers can be obtained through:

$$
\begin{aligned}
\text { Comm }_{\text {In-layer }}= & \sum_{p_{1}=1}^{P} \sum_{p_{2}=1}^{P} \sum_{d=1}^{D_{X}+D_{Y}} I_{p_{1}, p_{2}} \\
& \times \text { In-layer }\left(p_{1}, p_{2}\right)_{d} \times d .
\end{aligned}
$$

Similarly, layer-to-layer communication overhead can be expressed as a multiplication of communicating processors' communication intensity and layer-to-layer distances:

$$
\begin{aligned}
& \text { Comm }_{\text {Inter-layer }}=\sum_{p_{1}=1}^{P} \sum_{p_{2}=1}^{P} \sum_{l=1}^{L} I_{p_{1}, p_{2}} \\
& \times \text { Inter-layer }\left(p_{1}, p_{2}\right)_{l} \times l \text {. }
\end{aligned}
$$

Both Comm $_{\text {In-layer }}$ and Comm Inter-layer uses $I_{p_{1}, p_{2}}$ to express the affinity between two processors, which is multiplied with the distance given by $d$ or $l$.

Based on the above constraints, the objective function can be defined as:

$\min \mathrm{Comm}=\mathrm{Comm}_{\text {In-layer }}+\phi \mathrm{Comm}_{\text {Inter-layer }}$.

As expressed before, $\phi$ can be used as a knob to evaluate communication reduction versus reliability. In Section 5, $\phi$ constant's value and its effects are evaluated. From a pure communication reduction perspective this value will probably be much higher. However, if TSV usage is not preferred due to reliability concerns, $\phi$ parameter can be adjusted to reflect this. In the baseline implementation, $\phi$ parameter is conservatively set to 0.1 .

While the objective function does not consider performance specifically, it will indirectly optimize the performance by reducing the overall communication overheads. Note that, this performance improvement will also be limited with the $\phi$ constant. Moreover, additional constraints will be required for performance evaluation; for example, a constraint that captures simultaneous communication. Similarly, energy results can also be obtained with necessary constraints.

\section{Experiments}

Experimental evaluation is performed on parallelized arraybased applications. Parallelization and code optimizations are implemented through Stanford University Intermediate Format (SUIF). Benchmarks used in the experiments are shown in Table 2. Experiments are conducted by fastforwarding the first 1 billion instructions, and simulating the next 300 million instructions. Number of data accesses are shown in the fourth column of Table 2. As shown in Table 3, the default number of device layers is set two and a single layer is composed of 48 unit areas which can be assigned to NoC nodes. As explained before, in the base configuration, $\phi$ parameter is set to 0.1 , conservatively . The ILP solution times varied between $3 \mathrm{~min}$ and $7 \mathrm{~h}$, averaging on about $42 \mathrm{~min}$. Overall complexity of the proposed scheme is NPcomplete since it is based on ILP. However, when the offline nature of the proposed scheme is considered, the solution times are within tolerable ranges. Moreover, it is possible to generate a sub-optimal solution in cases of longer solution times, which usually tends to be very close to the optimal solution.

Experiments are conducted on four different execution models, namely, 2D-HM, 2D-HT, 3D-HM, and 3D-HT:

- 2D-HM: A single layer of 2D conventional NoC topology with homogeneous processors.
Table 2 Benchmark codes used in this study

\begin{tabular}{lllr}
\hline Benchmark & Source & Description & $\begin{array}{l}\text { Number of } \\
\text { data accesses }\end{array}$ \\
\hline 3step-log & DSPstone & Motion estimation & $91 \times 10^{6}$ \\
adi & Livermore & Alternate direction integration & $71 \times 10^{6}$ \\
ammp & Spec & Computational chemistry & $87 \times 10^{6}$ \\
equake & Spec & Seismic wave propagation sim. & $84 \times 10^{6}$ \\
mcf & Spec & Combinatorial optimization & $115 \times 10^{6}$ \\
mesa & Spec & 3D graphics library & $135 \times 10^{6}$ \\
vortex & Spec & Object-oriented database & $164 \times 10^{6}$ \\
vpr & Spec & FPGA circuit placement & $117 \times 10^{6}$ \\
\hline
\end{tabular}


Table 3 The default simulation parameters

\begin{tabular}{ll}
\hline Parameter & Value \\
\hline Types of processor cores & 4 \\
Number of blocks & 48 \\
Number of layers & 2 \\
Total storage capacity & $128 \mathrm{~KB}$ \\
Set associativity & 2 -way \\
Line size & 32 bytes \\
Number of lines per block & 90 \\
Temperature bound & $110{ }^{\circ} \mathrm{C}$ \\
Reliability $(\phi)$ & 0.1
\end{tabular}

- 2D-HT: Optimal placement of heterogeneous processors on a $2 \mathrm{D}$ grid using an ILP-based strategy. This uses the same optimization framework proposed so far, except it only considers a single layer that is the optimal placement scheme for 2D.

- 3D-HM: Homogeneous processors are distributed among a 3D stacked chip based on the communication requirements. Note that, this scheme also applies ILP-based approach and finds optimal placement.

- 3D-HT: Heterogeneous processor cores are placed on several layers optimally using the proposed ILP-based placement strategy. This scheme represents the optimal placement for 3D depending on the communication frequencies of nodes and the level of reliability.

Reliability-oriented data communication results normalized with respect to 2D-HM scheme based on two layers are given in Fig. 3. Using the default values given in Table 3, the average reduction in reliability-oriented data access costs for 2D-HT and 3D-HM are around 30 and $44 \%$, respectively. $3 \mathrm{D}-\mathrm{HT}$ reduces the communication further by $54 \%$ on average. 3D NoC reduces the global interconnect

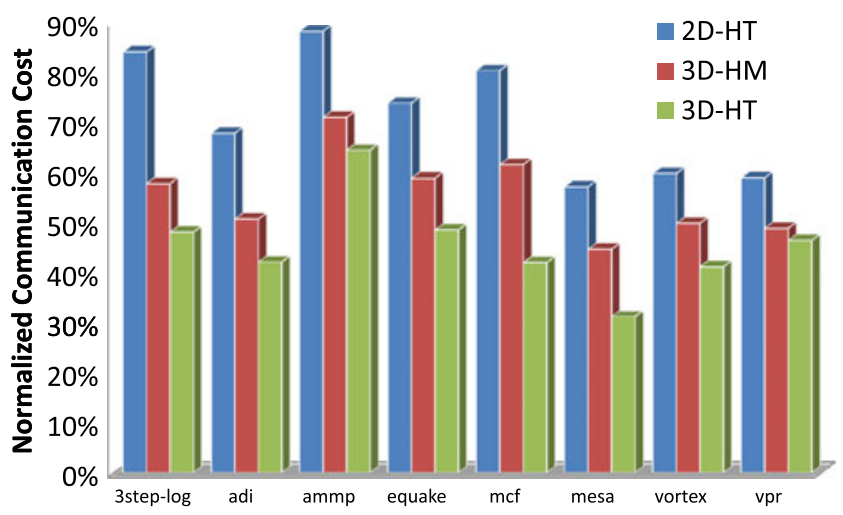

Fig. 3 Reliability-oriented data communication costs of 2D-HT, 3D$\mathrm{HM}$, and 3D-HT normalized with respect to 2D-HM

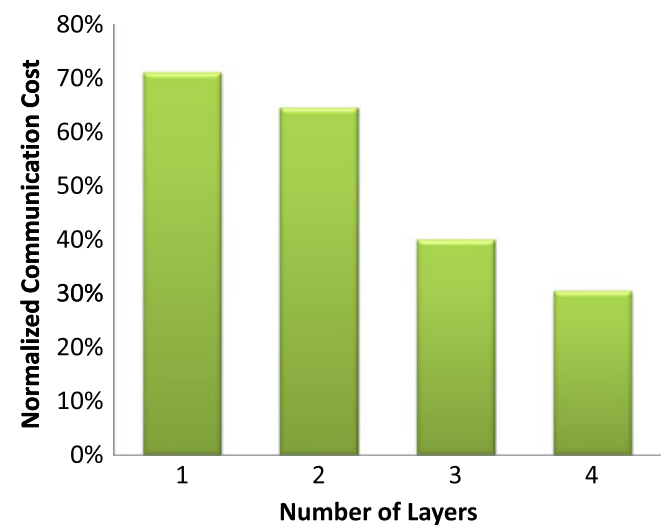

Fig. 4 Normalized reliability-communication costs with different number of layers (ammp)

length and improves overall communication while maintaining reliability. This is more pronounced with heterogeneous processors as there are more opportunities.

Recall that the number of 3D layers used were two. The bar-chart in Fig. 4 shows the normalized costs (with respect to those of the 2D-HM scheme) for the benchmark ammp with the different number of layers (the results with the original number of layers are also shown for convenience), ranging from 1 to 4 . Note that, the total storage capacity is kept constant for all these experiments and the only difference between two experiments is the number of layers and size of each layer. The number of layers and the corresponding number of blocks per layer for each topology tested are given in Table 4. One can see from these results that the effectiveness of the ILP-based approach increases with increasing number of layers. The main reason for this behavior is that adding more layers gives more flexibility to the proposed approach in placement.

In the next set of experiments, the effect of the $\phi$ parameter in communication cost savings is tested. As one can expect, savings increase with lower $\phi$ values. The main reason for this behavior is the reduction in the relative layer-to-layer communication cost, thereby increasing the flexibility on the vertical placement. On the other hand, from a reliability point of view, it is preferable to minimize the vertical communication on TSVs. Figure 5 shows the performance and reliability effects of the $\phi$ parameter.

Table 4 Different topologies used in experiments

\begin{tabular}{ll}
\hline $\begin{array}{l}\text { Number } \\
\text { of layers }\end{array}$ & $\begin{array}{l}\text { Number of blocks } \\
\text { per layer }\end{array}$ \\
\hline 1 & 48 \\
2 & 24 \\
3 & 16 \\
4 & 12
\end{tabular}




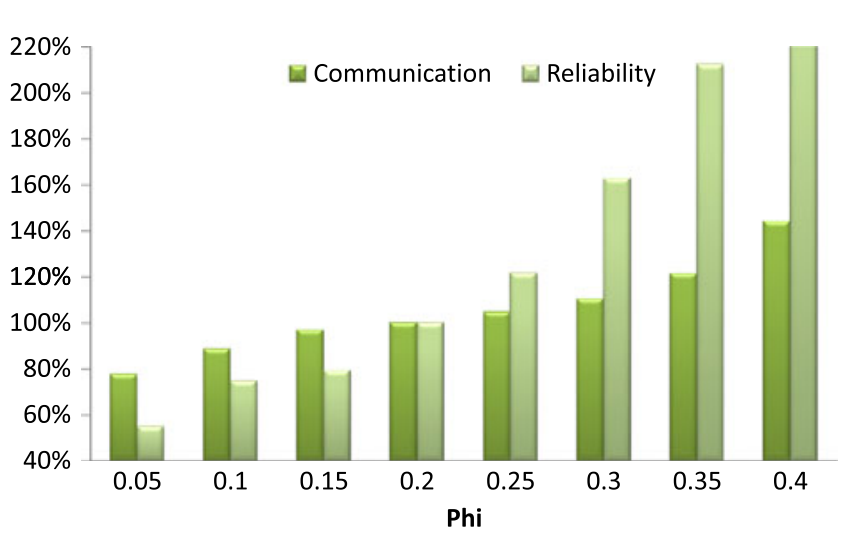

Fig. 5 Normalized reliability-communication costs under the different $\phi$ values (ammp)

As mentioned before, the default value of $\phi$ is 0.1 . Hence, all reliability and performance values are normalized with respect to $\phi=0.1$. As can be seen from the figure, when $\phi$ is increased, the normalized communication cost increases since the cost of transfer between layers is higher. Similarly, reliability of the communications also increases due to reduced usage of TSVs. Note that, reliability is measured using the amount of vertical communication cost which

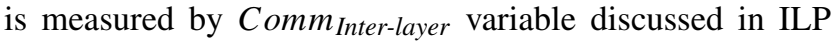
formulation.

In the last set of experiments, the impact of the temperature constraint on the savings is measured. Recall from Table 3 that the default temperature bound used in the experiments so far was $110{ }^{\circ} \mathrm{C}$. The bar-chart in Fig. 6 shows the normalized costs for the benchmark ammp with the different temperature bounds, ranging from 80 to $110{ }^{\circ} \mathrm{C}$. Note that, the values given in this figure are normalized with respect to the default 3D-HT case, where the best results are obtained. As can be seen from this figure, having a tighter temperature bound reduces savings beyond a certain point. The reason for this behavior is that decreasing the temperature bound also decreases the flexibility in processor core assignment.

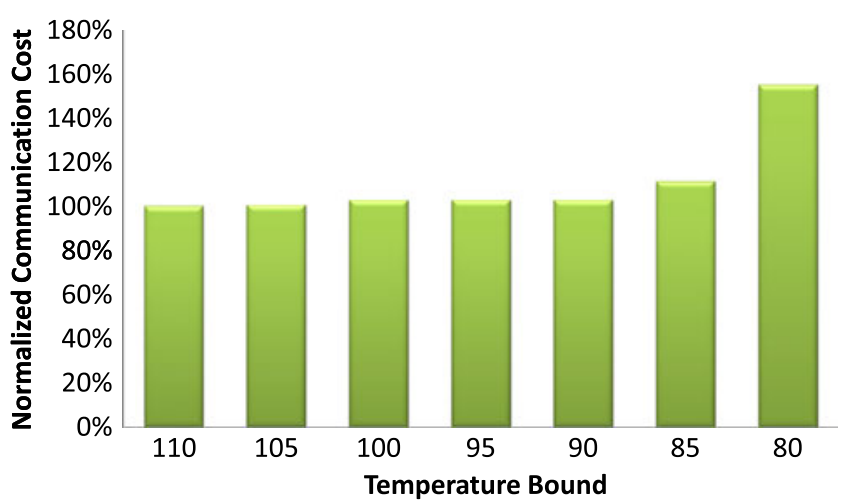

Fig. 6 Normalized reliability-communication costs under the different temperature bounds (ammp)
For this particular example, reducing the temperature bound below $80{ }^{\circ} \mathrm{C}$ did not return any feasible solution.

\section{Conclusion}

3D NoCs have been proposed to provide higher performance and lower power consumption by reducing the global interconnect length. However, reliability problem has become more important for 3D ICs with the shrinking technologies. This paper proposes an ILP-based near-optimal (if not optimal) 3D node mapping to maximize reliability while minimizing the communication costs. Experiments indicate that, through effective mapping, it is possible to achieve performance benefits while improving reliability. Although initial experiments are limited to few layers of 3D stacking, it is planned to increase the layers of 3D stacking and test with more complex structures.

Acknowledgments This research is supported in part by a grant from Turk Telekom under Grant Number 3015-04, and by a Marie Curie International Reintegration Grant within the 7th European Community Framework Programme.

\section{References}

1. Akasaka Y (1986) Three-dimensional IC trends. Proc IEEE 74(12):1703-1714

2. Alam SM, Troxel DE, Thompson CV (2004) Circuit and system level tools for thermal-aware reliability assessments of IC designs. Tech rep

3. Andry P, Sakuma K, Dang B, Maria J, Tsang C, Patel C, Wright S, Webb B, Sprogis E, Kang S, Polastre R, Horton R, Knickerbocker J (2007) 3D chip stacking technology with low-volume lead-free interconnections. In: Proceedings of 57th electronic components and technology conference, 2007. ECTC '07, pp 627-632

4. Davis W, Wilson J, Mick S, Xu J, Hua H, Mineo C, Sule A, Steer M, Franzon P (2005) Demystifying 3D ICs: the pros and cons of going vertical. IEEE Des Test Comput 22(6):498-510

5. International Technology Roadmap for Semiconductors. http:// public.itrs.net/

6. Li F, Nicopoulos C, Richardson T, Xie Y, Narayanan V, Kandemir $M$ (2006) Design and management of 3D chip multiprocessors using network-in-memory. In: 33rd international symposium on computer architecture, 2006. ISCA '06, pp 130-141

7. Loi I, Angiolini F, Benini L (2008) Developing mesochronous synchronizers to enable 3D NoCs. In: Design, automation and test in Europe, 2008. DATE '08, pp 1414-1419

8. Malta D, Gregory C, Lueck M, Temple D, Krause M, Altmann F, Petzold M, Weatherspoon M, Miller J (2011) Characterization of thermo-mechanical stress and reliability issues for $\mathrm{Cu}$-filled TSVs. In: IEEE 61st electronic components and technology conference (ECTC), 2011, pp 1815-1821

9. Minas N, De Wolf I, Marinissen E, Stucchi M, Oprins H, Mercha A, Van der Plaas G, Velenis D, Marchal P (2010) 3D integration: circuit design, test, and reliability challenges. In: IEEE 16th international on-line testing symposium (IOLTS), 2010, p 217 
10. Minz J, Wong E, Lim SK (2005) Reliability-aware floorplanning for 3D circuits. In: Proceedings of IEEE international SOC conference, 2005, pp 81-82

11. Murata H, Fujiyoshi K, Nakatake S, Kajitani Y (1995) Rectanglepacking-based module placement. In: IEEE/ACM international conference on computer-aided design, 1995. ICCAD-95. Digest of technical papers, pp 472-479

12. Dash Optimization Ltd. (2005) Xpress-MP optimizer reference manual. Nothants, UK. http://www.dashoptimization.com

13. Ozturk O, Wang F, Kandemir M, Xie Y (2006) Optimal topology exploration for application-specific 3D architectures. In: Asia and South Pacific conference on design automation 2006

14. Pavlidis V, Friedman E (2007) 3-D topologies for networkson-chip. IEEE Trans Very Large Scale Integr (VLSI) Syst 15(10):1081-1090

15. Sakuma K (2011) Development trend of three-dimensional (3D) integration technology. IEEJ Trans Sens Micromachines 131:1925

16. Selvanayagam C, Lau J, Zhang X, Seah S, Vaidyanathan K, Chai $\mathrm{T}$ (2008) Nonlinear thermal stress/strain analyses of copper filled TSV (through silicon via) and their flip-chip microbumps. In: 58th electronic components and technology conference, 2008, pp 1073-1081

17. Shayan A, Hu X, Peng H, Cheng CK, Yu W, Popovich M, Toms T, Chen X (2009) Reliability aware through silicon via planning for 3D stacked ICs. In: design, automation test in Europe conference exhibition, 2009. DATE '09, pp 288-291
18. Topol AW, Tulipe DCL, Shi L, Frank DJ, Bernstein K, Steen SE, Kumar A, Singco GU, Young AM, Guarini KW, Ieong M (2006) Three-dimensional integrated circuits. IBM J Res Dev 50(4.5):491-506

19. Zhao X, Scheuermann M, Lim SK (2012) Analysis of DC current crowding in through-silicon-vias and its impact on power integrity in 3D ICs. In: 49th ACM/EDAC/IEEE design automation conference (DAC), 2012, pp 157-162

Ismail Akturk is an M.S. Student in the Department of Computer Engineering at Bilkent University, Turkey. Before joining Bilkent, he was a research assistant in Center for Computation and Technology at Louisiana State University. He got his first M.S. degree in Electrical Engineering from the Department of Electrical and Computer Engineering at Louisiana State University in 2009, and his B.S degree from Dogus University, Istanbul, Turkey in 2007.

Ozcan Ozturk is an Assistant Professor in the Department of Computer Engineering at Bilkent University. Prior to joining Bilkent, he worked as a software optimization engineer in Cellular and Handheld Group at Intel (Marvell). He received his PhD from The Pennsylvania State University, the M.S. degree from University Of Florida, and received the B.Sc. degree from Bogazici University, all in Computer Engineering. His research interests are in the areas of chip multiprocessing, computer architecture, manycore architectures, and parallel processing. He is a recipient of 2009 IBM Faculty Award and 2009 Marie Curie Fellowship from European Commission. 